Peer

Analytical Chemistry
Submitted 26 July 2021

Accepted 4 November 2021

Published 23 November 2021

Corresponding author

Jonas Sundberg, jonsun@dtu.dk

Academic editor

Oliver Jones

Additional Information and Declarations can be found on page 14

DOI 10.7717/peerj-achem.12

Copyright

2021 Huynh et al.

Distributed under

Creative Commons CC-BY 4.0

OPEN ACCESS

\section{Extended characterization of petroleum aromatics using off-line LC-GC-MS}

\author{
Khoa Huynh, Annette E. Jensen and Jonas Sundberg \\ Danish Hydrocarbon Research and Technology Centre, Technical University of Denmark, Kgs. Lyngby, \\ Denmark
}

\section{ABSTRACT}

Characterization of crude oil remains a challenge for analytical chemists. With the development of multi-dimensional chromatography and high-resolution mass spectrometry, an impressive number of compounds can be identified in a single sample. However, the large diversity in structure and abundance makes it difficult to obtain full compound coverage. Sample preparation methods such as solid-phase extraction and SARA-type separations are used to fractionate oil into compound classes. However, the molecular diversity within each fraction is still highly complex. Thus, in the routine analysis, only a small part of the chemical space is typically characterized. Obtaining a more detailed composition of crude oil is important for production, processing and environmental aspects. We have developed a highresolution fractionation method for isolation and preconcentration of trace aromatics, including oxygenated and nitrogen-containing species. The method is based on semipreparative liquid chromatography. This yields high selectivity and efficiency with separation based on aromaticity, ring size and connectivity. By the separation of the more abundant aromatics, i.e., monoaromatics and naphthalenes, trace species were isolated and enriched. This enabled the identification of features not detectable by routine methods. We demonstrate the applicability by fractionation and subsequent GC-MS analysis of 14 crude oils sourced from the North Sea. The number of tentatively identified compounds increased by approximately 60 to $150 \%$ compared to solidphase extraction and GC $\times$ GC-MS. Furthermore, the method was used to successfully identify an extended set of heteroatom-containing aromatics (e.g., amines, ketones). The method is not intended to replace traditional sample preparation techniques or multi-dimensional chromatography but acts as a complementary tool. An in-depth comparison to routine characterization techniques is presented concerning advantages and disadvantages.

Subjects Hyphenated Techniques, Mass Spectrometry, Sample Handling

Keywords Petroleomics, PAHs, GC-MS, Crude oil

\section{INTRODUCTION}

The use of petroleum as a feedstock for energy production is declining. However, certain critical functions cannot safely be replaced by renewable energy (International Energy Agency, IEA)(2021). Secondly, petroleum is a fundamental feedstock for the production of a large number of chemical starting materials (Aftalion, 2001; Yadav, Yadav \& Patankar, 2020). Therefore, reducing the environmental impact of oil production is an important goal. This requires a better understanding of petroleum on the molecular level. Crude 
oil is a complex mixture of saturated and aromatic hydrocarbons with a smaller fraction of heteroatom-containing compounds, i.e., the resins and asphaltenes. The molecular distribution typically ranges from 16 to $1,000 \mathrm{amu}$ (Marshall \& Rodgers, 2008). The number of unique compounds is extensive and more than 240,000 molecular species have been resolved in a single sample (Krajewski, Rodgers \& Marshall, 2017; Palacio Lozano et al., 2019). Due to this complexity, a large portion of the petroleum chemical space is structurally unknown.

We have previously looked at the resins fraction (i.e., polar heteroatom-containing species) of North Sea oils (Sundberg \& Feilberg, 2020). Herein, we extend our work with a focus on aromatics. Within this fraction, the dominant species (in terms of abundance) are monoaromatic followed by a smaller amount of polycyclic aromatic hydrocarbons (PAHs) (Requejo et al., 1996; Wei et al., 2018). The PAHs class is dominated by smaller (2 to 3 rings) PAHs, with larger species (e.g., chrysene, coronene) being present at trace levels. It also contains small amounts of heteroatomic-containing ring structures (Mössner \& Wise, 1999; Zhang et al., 2018; Carvalho Dias et al., 2020). Due to their toxicity, PAHs have been extensively studied (Lawal, 2017). A large focus has been on the 16 priority pollutants PAHs defined by the US Environmental Protection Agency (Keith, 2015). However, this list is not representative of crude oils which contain a more structurally diverse PAH set (Andersson \& Achten, 2015; Stout et al., 2015, p. 16). Low molecular weight PAHs are susceptible to weathering, primarily by volatilization, whereas high molecular weight aromatics are more resilient (John, Han \& Clement, 2016). Therefore, these are useful targets for oil-oil and oil-source correlation and spill identification and environmental monitoring (Pampanin \& Sydnes, 2017; Poulsen et al., 2018).

Comprehensive identification of the aromatics is challenging due to the large concentration variance. Traditionally, petroleum analysis is based on pre-fractionation using silica chromatography or solid-phase extraction (SPE) cartridges followed by GC-MS (Wang, Fingas \& Li, 1994; Alzaga et al., 2004; Pillai et al., 2005; Gilgenast et al., 2011). SPE is a low-efficiency separation technique, based on chemical selectivity. This allows crude isolation of the aromatics fraction, but not separation of the compounds within it (Fig. 1). Thus, an aromatic fraction obtained by SPE contains both the benzenes, naphthalenes and larger rings. Here, a typical crude oil will have a high abundance of monoaromatics, with diminishing concentrations with increasing ring size. The appropriate GC on-column concentration of the naphthalenes typically results in the larger ring systems being below the limit of detection (LOD). By increasing concentration to push trace aromatics above the LOD, both the column and detector will be saturated by the more abundant compounds. This leads to high background levels which affect quantitation and may obscure mass spectra complicating structural identification of unknowns (Zhao et al., 2014; Wilton, Wise $\&$ Robbat, 2017). Furthermore, the poor resolution of SPE often leads to an overlap between the saturated and aromatic hydrocarbons, which interferes with subsequent analysis. Thus, although SPE is efficient for routine applications, a large portion of the sample remains undetected. Comprehensive multi-dimensional chromatography $(\mathrm{GC} \times \mathrm{GC})$ is often used as an alternative to simplify or remove the need for sample pre-fractionation (Nizio, McGinitie \& Harynuk, 2012; Jennerwein et al., 2014; Stilo et al., 2021). However, it does not 

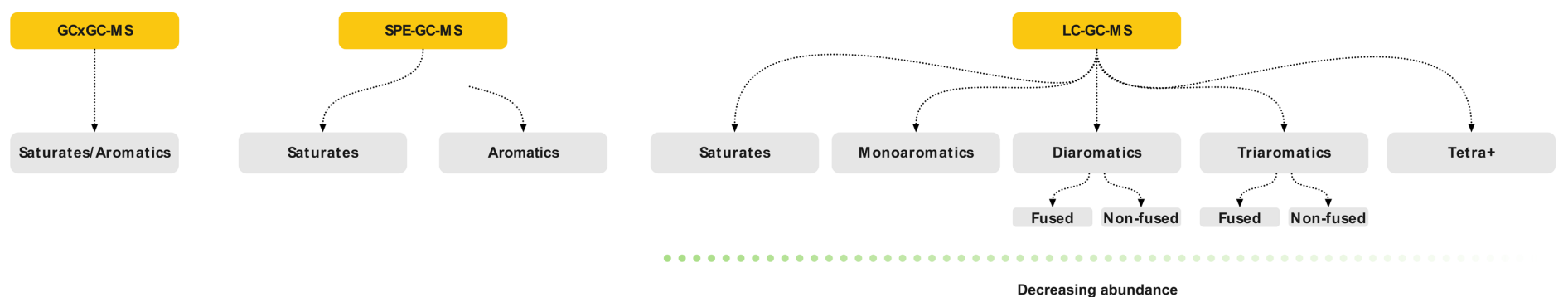

Figure 1 Schematic of the three sample preparation and analytical strategies discussed in the paper showing the extent of fractionation possible. Grey boxes indicate the individual samples that are characterized by mass spectrometry.

Full-size DOI: $10.7717 /$ peerjachem.12/fig-1

solve the issue with variable abundance and column/detector overload. Thus, a complete qualitative and explorative oil analysis requires a more selective sample-prefractionation method.

Herein, we present a high-performance liquid-chromatography (HPLC) method for the automated high-resolution fractionation of crude oil using commercially available columns. The method can resolve aromatics based on ring size and connectivity, i.e., fused and non-fused rings (e.g., naphthalene versus biphenyl). The fractions may be diluted or concentrated, depending on the target, for subsequent analysis and can thus be used to concentrate trace species. The method is easily modified to selectively collect only fractions of interest, and the aromatics may be collected either as one or several fractions. We demonstrate the method's applicability by fractionation of fourteen crude oils with subsequent GC-MS analysis. The method is compared to data obtained using SPE and GC $\times$ GC-MS. We demonstrate how it's especially suitable for the analysis and identification of trace aromatics by the successful tentative identification of several compounds not observed using comparable methods.

\section{MATERIALS \& METHODS Chemicals and reagents}

Chloroform, dichloromethane, $n$-hexane, deuterated standards and model compounds (ethylbenzene, naphthalene, biphenyl, phenanthrene, 1-benzylnaphthalene and chrysene) were purchased from Sigma Aldrich and used as received.

\section{Samples}

Fourteen crude oils sampled from producing fields in the Danish region of the North Sea were obtained from Mærsk Oil (now Total E\&P). The samples were received in metal containers (jerrycans) and transferred to glass bottles upon arrival. The samples were stored at room temperature protected from light.

\section{Sample preparation Solid-phase extraction}

Crude oil $(10 \mu \mathrm{L})$ was combined with $100 \mu \mathrm{L}$ of a solution containing alkane internal standards (decane-D22, hexadecane-D34 and eicosane-D42, $400 \mu \mathrm{g} / \mathrm{mL}$ in $n$-hexane), 50 
$\mu \mathrm{L}$ of PAH internal standards (naphthalene-D8, phenanthrene-D10, acenaphthene-D10, chrysene-D12 and perylene-D12, $30 \mu \mathrm{g} / \mathrm{mL}$ in $n$-hexane) and further diluted with $n$-hexane $(840 \mu \mathrm{L})$. A solid-phase extraction column (Phenomenex EPH Strata, $200 \mu \mathrm{m}, 70 \AA, 500$ $\mathrm{mg} /$ three $\mathrm{mL}$ ) was cleaned and conditioned by $\mathrm{CH}_{2} \mathrm{Cl}_{2}(3 \times 1 \mathrm{~mL})$ followed by $n$-hexane $(3 \times 1 \mathrm{~mL}) .100 \mu \mathrm{L}$ of oil solution was carefully applied to the column and was allowed to settle for $5 \mathrm{~min}$. Saturated hydrocarbons were eluted into one fraction with three portions of $n$-hexane $(3 \times 600 \mu \mathrm{L})$. Aromatic hydrocarbons were eluted using dichloromethane $(1 \times 1,800 \mu \mathrm{L})$. The solvent level of each fraction was reduced to $500 \mu \mathrm{L}$ under a gentle stream of nitrogen without applied heating to avoid losses of volatile components.

\section{Liquid chromatography fractionation}

Fractionation of crude oil was carried out on a Dionex UltiMate 3000 HPLC equipped with a DAD-3000 diode array, a RefractoMax RI-521 refractive index (RI) detector and an AFC-3000 fraction collector. The system was fitted with one six-port/two-way and one ten-port/two-way port to enable selective backflush of the primary column. A Thermo Scientific Hypersil Gold $\mathrm{NH}_{2}$ (4.6 mm i.d., $3 \mu \mathrm{m}, 150 \mathrm{~mm}$ ) and a Hypersil Silica (4.6 mm i.d., $3 \mu \mathrm{m}, 150 \mathrm{~mm}$ ) were connected in series. The sample manager was kept at $20^{\circ} \mathrm{C}$ and the column oven at $30^{\circ} \mathrm{C}$. The injection volume was $50 \mu \mathrm{L}$.

Samples were diluted at 1:2000 in $n$-hexane and stored at $-20^{\circ} \mathrm{C}$ for $24 \mathrm{~h}$ to precipitate asphaltenes. The samples were centrifuged and an aliquot of the mother liquor was carefully transferred to an autosampler vial for analysis. Separation of saturates and aromatics was achieved via isocratic $n$-hexane elution during which $30 \mathrm{~s}$ wide fractions were collected. After elution of aromatics, the primary column was rinsed using a backflush gradient from $n$-hexane to 1:1 2-propanol:chloroform. The collected fractions were diluted (saturates, mono- and di-aromatics) or concentrated (tri-aromatics and larger) for analysis on GC-MS. For enrichment experiments, consecutive fractionations (typically 3 to 6) were performed with pooling of the eluents followed by solvent reduction under a gentle stream of $\mathrm{N}_{2}$ at $30{ }^{\circ} \mathrm{C}$.

\section{Analytical methods GC-MS}

GC-MS data were recorded using an Agilent 5977B GC-MSD as follows; $250{ }^{\circ} \mathrm{C}$ inlet, $320^{\circ} \mathrm{C}$ transfer line, splitless injection $(1 \mu \mathrm{L})$, Agilent DB-5MS ( $30 \mathrm{~m}, 0.25 \mathrm{~mm}$ i.d., 0.25 $\mu \mathrm{m})$. The oven temperature gradient was programmed as follows; 50 (1 min. hold-time) $320^{\circ} \mathrm{C}\left(8 \mathrm{~min}\right.$ hold-time, $10^{\circ} \mathrm{C} / \mathrm{min}$.), helium carrier gas at $1.5 \mathrm{~mL} / \mathrm{min}$. in constant flow mode.

GC $\times$ GC-MS data were recorded using an Agilent 7200B GC-QTOF equipped with a Zoex ZX-2 thermal modulator (Zoex Corporation, Houston, TX, USA) as follows; $250^{\circ} \mathrm{C}$ inlet, $320^{\circ} \mathrm{C}$ transfer line, splitless injection $(1 \mu \mathrm{L})$, Agilent DB-5MS UI (1D, $30 \mathrm{~m}, 0.25 \mathrm{~mm}$ i.d., $0.25 \mu \mathrm{m} \mathrm{df}$ ) and a Restek Rxi-17Sil MS (2D, $2 \mathrm{~m}, 0.18 \mathrm{~mm}$ i.d., $0.18 \mu \mathrm{m} \mathrm{df}$ ) capillary columns connected using a SilTite $\mu$-union. The oven was temperature programmed as follows; 50 (1 min hold-time) $-320^{\circ} \mathrm{C}\left(3{ }^{\circ} \mathrm{C} / \mathrm{min}\right.$. $)$, helium carrier gas at one $\mathrm{mL} / \mathrm{min}$. in constant flow mode. The modulation period was set to $6 \mathrm{~s}$ with a $400 \mathrm{~ms}$ hot-jet duration. 


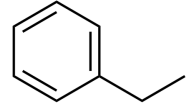

Ethylbenzene

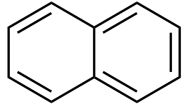

Naphthalene

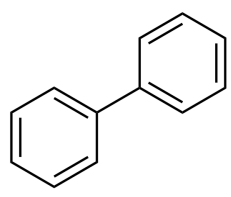

Biphenyl

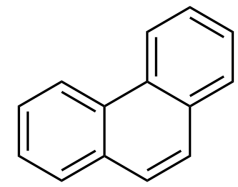

Phenanthrene<smiles>c1ccc(Cc2cccc3ccccc23)cc1</smiles>

1-Benzylnaphthalene<smiles>c1ccc2c(c1)ccc1c3ccccc3ccc21</smiles>

Chrysene

Figure 2 Molecular structure of the model compounds used for the HPLC method development and validation.

Full-size DOI: 10.7717/peerjachem.12/fig-2

\section{Data processing}

Data were screened using Masshunter Qualitative Navigator (Agilent, B.08.00). Peak detection and compound identification were performed using MassHunter Unknowns Analysis (Agilent, B.09.00) and the NIST Standard Reference Database (1A v17, Gaithersburg, MD, USA). Feature tables were exported as CSV files and imported into a Jupyter Notebook for further processing using the Python scripting language. Duplicates based on the CAS number were removed from the feature tables. All compounds containing silica and halogens were removed. The double-bond equivalent values were calculated for each compound and all features with a DBE of less than 4 were excluded. Finally, experimental and literature retention indices (RI) were compared with flagging of all compounds where the difference was larger than 50 units.

\section{RESULTS AND DISCUSSION}

\section{Method development}

The objective of the method was to (1) separate saturates and aromatics and (2) intra-class separation of the aromatics with enrichment capabilities. A dual-column setup using normal phase analytical LC-columns provided the required selectivity and efficiency. The primary column (Thermo Scientific Hypersil Gold $\mathrm{NH}_{2}$ ) acted as a retainer for polar components, whereas a secondary pure silica-based column (Thermo Scientific Hypersil Silica) was required for the separation of saturated and aromatic hydrocarbons. The separation was optimized using six model compounds commonly found in crude oil (Fig. 2). An isocratic $n$-hexane elution yielded separation of saturated and mono-aromatic hydrocarbons, as well as separation of polycyclic aromatics based on ring size and connectivity (Fig. 3).

The fraction collector was programmed to collect $30 \mathrm{~s}$ wide fractions based on the peak widths of the model compounds. At this fraction width, we observed only a minor overlap of fractions with co-elution of the most abundant components. A reduction of the fraction width can be set if a higher peak purity is required. The cost is a slight loss of recovery. After elution of the last aromatics as observed by UV/Vis, the flow path was selectively reversed for the primary column. The column was then rinsed using a gradient from 100\% $n$-hexane to 50:50 chloroform:2-propanol. This effectively removed the adsorbed resins on the amide column. The fraction collector is within the flow path during all stages of chromatography and the resins may therefore be isolated for further analysis 


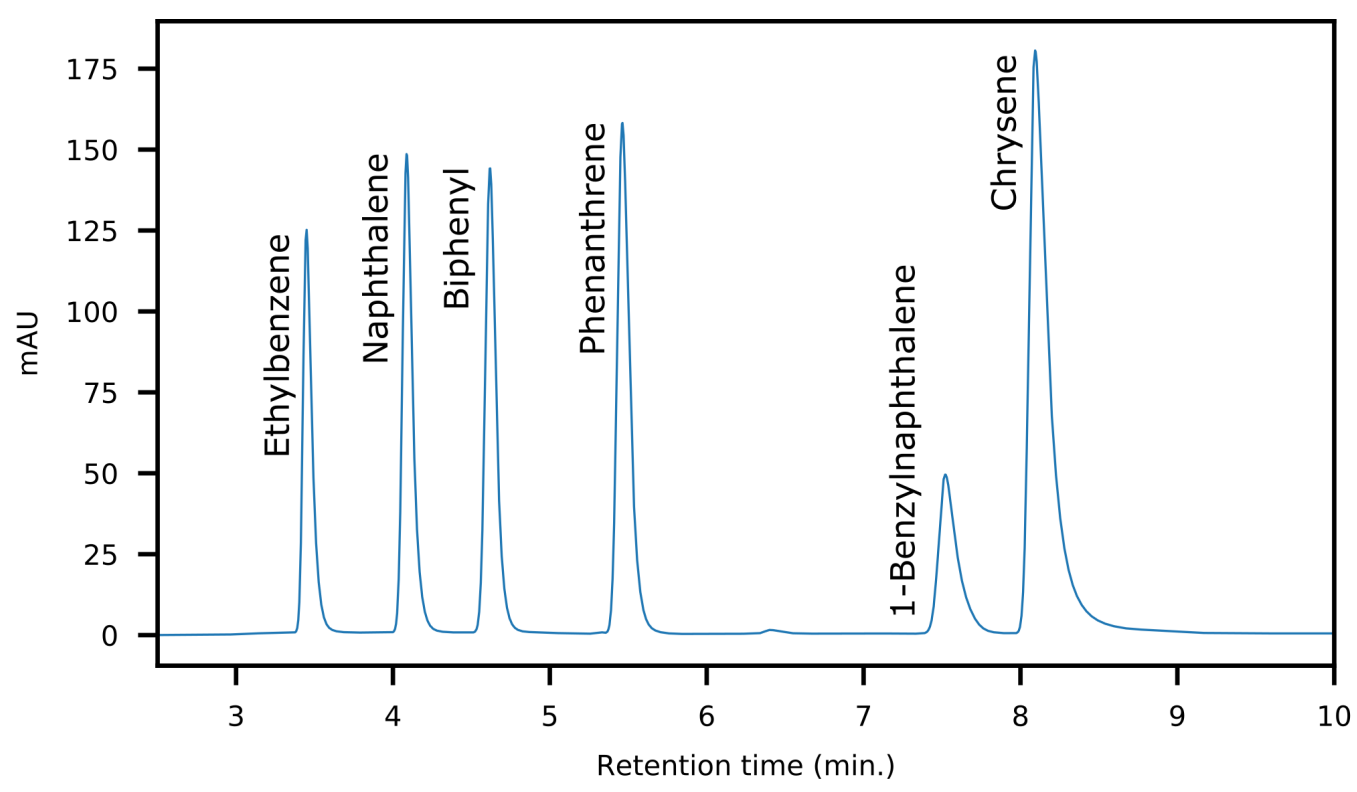

Figure 3 HPLC-Chromatogram showing the elution order and separation of the model compounds used for method development.

Full-size DOI: 10.7717/peerjachem.12/fig-3

(Sundberg \& Feilberg, 2020). The final step is a re-equilibration of the whole system by a return to isocratic $n$-hexane and flushing at an increased flow rate to remove the polar solvents from the flow path. Improper re-equilibration resulted in a severe loss of retention in subsequent fractionations due to the adsorption of 2-isopropanol on the silica phase.

Recovery values were calculated using the model compounds by comparison of peak areas obtained on GC-MS from LC-fractions compared to direct analysis of the standards (Table 1). Three analytes have recovery values slightly above $100 \%$. This is likely due to a discrepancy between programmed and real injection volume on the HPLC auto-sampler. In contrast, the recovery is less than $90 \%$ for chrysene. For this compound, we observe peak broadening due to the high capacity factor. As the fraction collection width is static during the full run, the low recovery is attributed to the peak being wider than the collection width. To evaluate the reproducibility of complex samples, a single oil was fractionated three consecutive times. Each fraction was analyzed on GC-MS and the relative standard deviation was determined from peak areas. 1,2,4-Trimethylbenzene, naphthalene and phenanthrene gave 4.6,8.1 and $6.4 \%$ respectively. The results are similar to those obtained using a model mixture. This shows that the method performs consistently in the presence of a highly complex oil matrix. Furthermore, the method successfully removes interferences and yields a high signal-to-noise ratio for the target analytes in each fraction (Fig. 4).

\section{Applicability in crude oil analyses}

The applicability of the method was demonstrated by fractionation and analysis of 14 crude oils. The oil samples were sourced from producing fields in the Danish region of the North Sea. Crude oils from this region typically have an aromatics content of 25-30\%, of which the majority are BTEX-type monoaromatics (benzene, toluene, ethylbenzene, xylene) with 
Table 1 Recovery calculations based on integrated area as determined by GC-MS for pure model compounds and those isolated using HPLC-fractionation.

\begin{tabular}{|c|c|c|c|}
\hline Model compounds & $\begin{array}{l}\text { Area, STD GC-MS } \\
(\% \mathrm{RSD}, N=6)\end{array}$ & $\begin{array}{l}\text { Area, STD LC-GC-MS } \\
(\% \mathrm{RSD}, N=6)\end{array}$ & Recovery (\%) \\
\hline Naphthalene & $3344437(1.0 \%)$ & $3566196(5.7 \%)$ & 106.6 \\
\hline Biphenyl & $1525309(1.6 \%)$ & $1627554(2.1 \%)$ & 106.7 \\
\hline Phenanthrene & $1145132(3.1 \%)$ & $1162530(4.7 \%)$ & 101.5 \\
\hline 1-Benzylnaphthalene & $599597(4.4 \%)$ & $592272(3.0 \%)$ & 98.8 \\
\hline Chrysene & $220091(5.1 \%)$ & $195914(2.8 \%)$ & 89.0 \\
\hline Crude oil compounds & $\begin{array}{l}\text { Area, SPE-GC-MS } \\
(\% \mathrm{RSD}, N=3)^{*}\end{array}$ & $\begin{array}{l}\text { Area,oil LC-GC-MS } \\
(\% \mathrm{RSD}, N=3)^{*}\end{array}$ & \\
\hline 1,2,4-Trimethylbenzene & $29538362(17.6 \%)$ & $12638320(4.6 \%)$ & \\
\hline Naphthalene & $20202722(18.0 \%)$ & $5153076(8.1 \%)$ & \\
\hline Phenanthrene & $9239818(17.4 \%)$ & $3478436(6.4 \%)$ & \\
\hline
\end{tabular}

a continuous decrease in abundance with increasing ring size (Sundberg \& Feilberg, 2020). The primary fraction is the saturated hydrocarbons followed by the resins (up to 5\%) with only traces of asphaltenes. This is evident from the fractionation, where a typical dilution factor of 50/20 had to be applied to the saturated and monoaromatic fractions respectively (Table 2). The fractions containing larger aromatics were analyzed either undiluted or concentrated by solvent reduction.

The first fraction contains the paraffins and naphthenes and is poorly retained on the primary LC-column (Fig. 5). The second silica column is required to separate them from the monoaromatics, which elute as the second fraction (Fig. 6). The third and fourth fractions contain diaromatic species, with the latter non-fused ring systems (e.g., naphthalene versus biphenyl). Fractions 5 and 6 contain the triaromatics (e.g., phenanthrene versus 1-phenylnaphthalene). Here, the abundance starts to diminish and the sixth fraction had to be concentrated for subsequent GC-MS analysis. Fractions 7 and above contain larger ring systems, e.g., chrysene, perylene. These fractions are less well-defined, likely because compounds eluting within this retention range are fewer in number and present in trace amounts. We also observed a slight loss of resolution, with minor overlap and crosscontamination. This is a result of two things; (1) diffusion and peak broadening during the liquid chromatography (2) collection of low abundance (undiluted/concentrated) fraction after a high abundance (diluted) fraction. If higher purity peaks are required the fraction collection width can be reduced. Attempts to concentrate fractions 9 and later were not successful and the gas chromatograms were dominated by background contaminants likely originating from the solvents, HPLC-tubing and glassware (e.g., siloxanes, surfactants).

\section{Performance comparison with SPE-GC-MS and GC x GC-MS}

Solid-phase extraction of crude oil into its saturated and aromatic fraction is a wellestablished sample preparation method. The physical properties of SPE adsorbents (large particle size, low mass loadings) result in limited separation power (Berrueta, Gallo \& Vicente, 1995; Buszewski \& Szultka, 2012). Thus, the technique is mainly applicable for the 

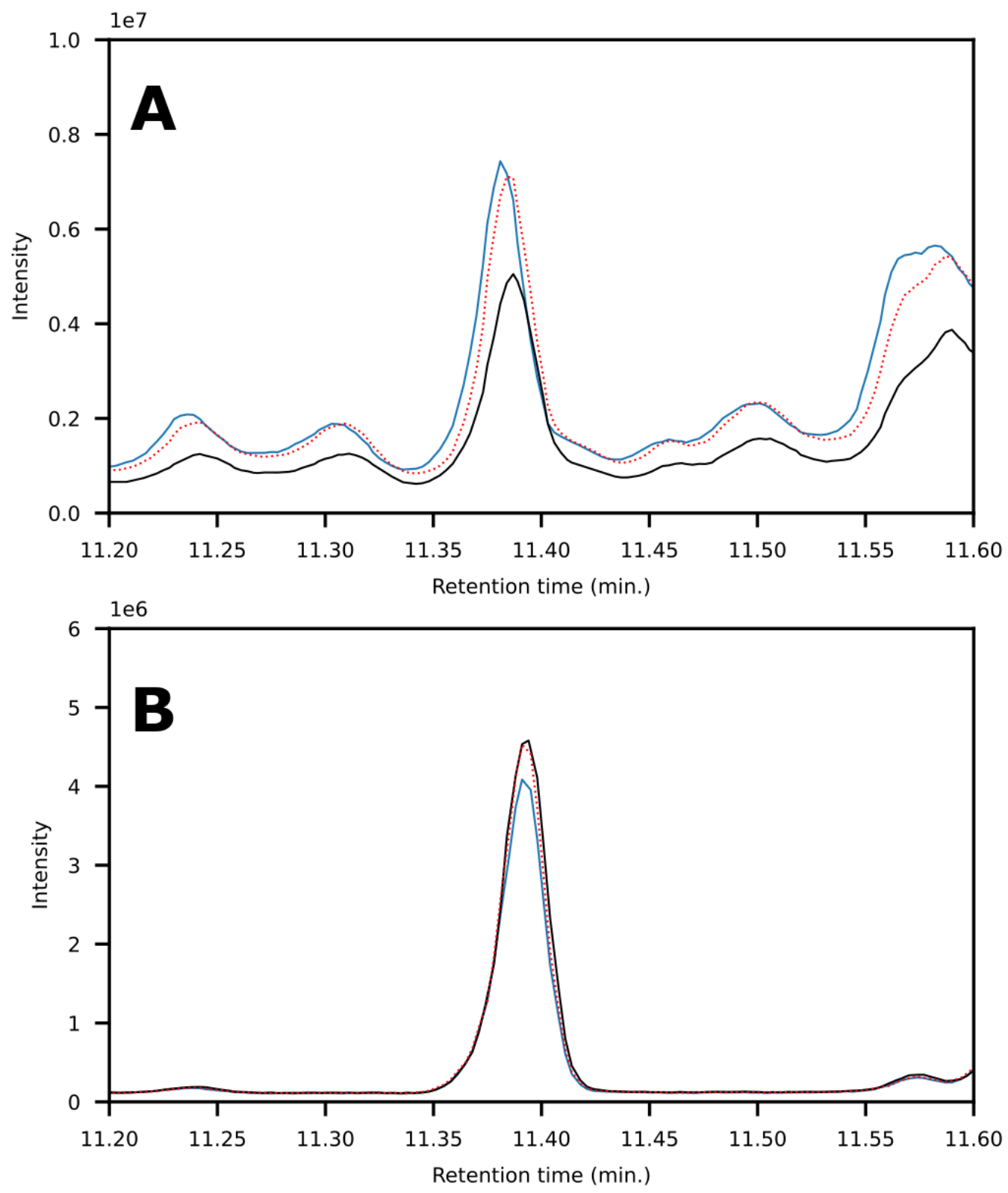

Figure 4 Chromatogram showing the peak corresponding to phenanthrene obtained via SPEextractions (A) as compared to LC-fractionation (B). The sample was fractionated using each method in triplicate to determine reproducibility. Signal intensity variance between (A) and (B) is due to different dilution factors (on-column).

Full-size DOI: 10.7717/peerjachem.12/fig-4

crude separation of different compound classes. It does not provide sufficient resolution to separate closely related compounds within subfractions. To compare our method to SPE we fractionated each oil using a Phenomenex Strata EPH ( $200 \mu \mathrm{m}, 70 \AA, 500 \mathrm{mg} /$ three $\mathrm{mL})$. The cartridge contains a proprietary phase specifically developed to separate hydrocarbon fractions (Countryman, Kelly \& Garriques, 2005). 


\begin{tabular}{lll}
\hline Table 2 & Summary & description of the major constituents of each fraction. \\
Fraction & Main composition & Comment \\
\hline 1 & Saturated hydrocarbons & High concentration; diluted for GC-MS. \\
2 & Mono-aromatics & High concentration; diluted for GC-MS. \\
3 & Di-aromatics & Medium concentration; diluted for GC-MS. \\
4 & Non-fused di-aromatics & Medium concentration; diluted for GC-MS. \\
5 & Tri-aromatics & Low concentration; undiluted for GC-MS. \\
6 & Non-fused tri-aromatics & Trace concentrations; concentrated for GC-MS. \\
$7+8$ & Misc. tetra-aromatics & Trace concentrations; concentrated for GC-MS. \\
\hline
\end{tabular}

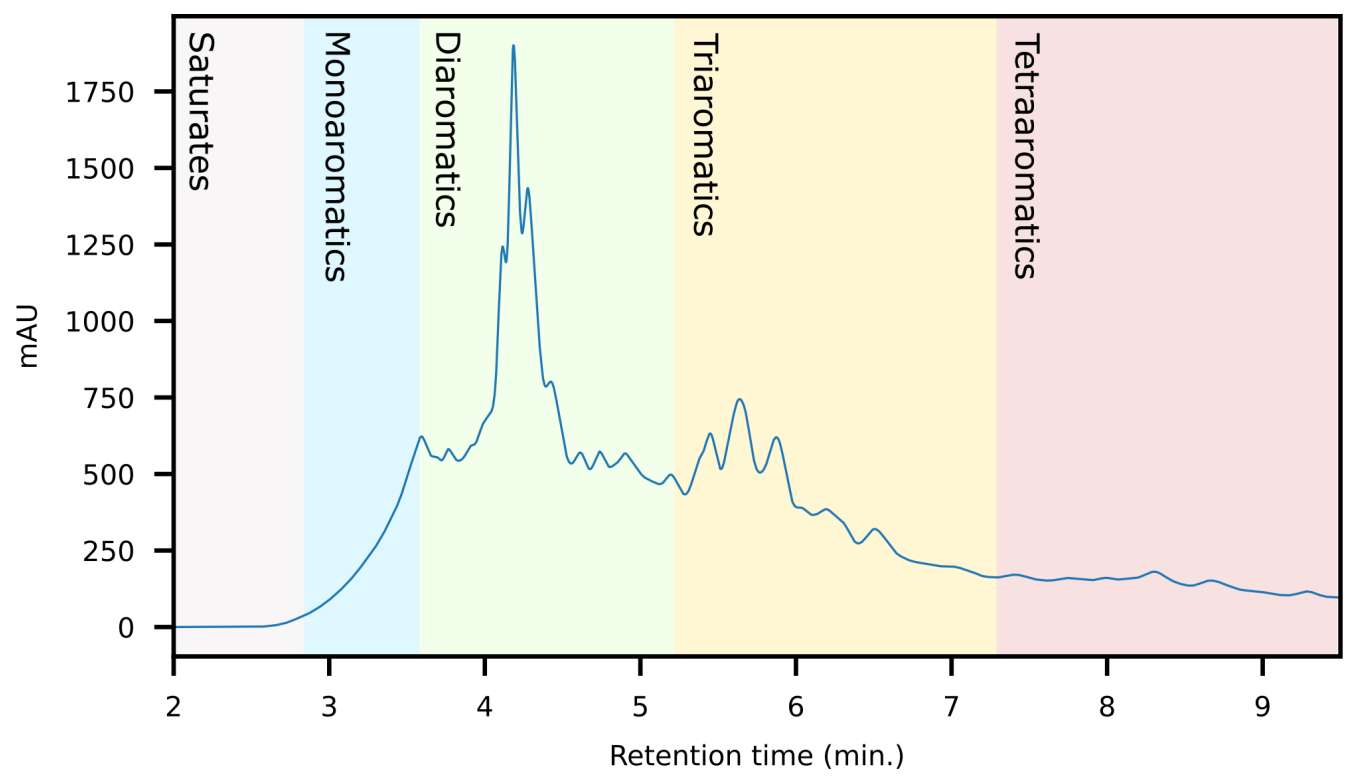

Figure 5 HPLC-Chromatogram (with UV response measured at $272 \mathrm{~nm}$ ) of a representative crude oil showing the approximate retention regions of the different aromatic classes.

Full-size DOI: 10.7717/peerjachem.12/fig-5

In terms of spectral quality, an approximately 10-fold reduction in background noise is observed in the LC fractions as compared to SPE. A comparison of the extracted mass spectra for the peak corresponding to 1,3-dimethylpyrene is presented in Fig. 7. Selected ion monitoring can be used to reduce background interferences for target species but results in loss of spectral detail for qualitative analysis. Furthermore, when using low-resolution instruments, i.e., single quadrupole MS, there is a large risk of overlap in complex samples (Rosenthal, 1982; (Davis \& Giddings, 1983). The reduction in background noise improved library matching, especially for analytes present at trace levels.

To evaluate identification performance, peak picking and library matching were carried out using MassHunter Unknowns Analysis and the NIST mass spectral library. The match factor limit was set to 700. The number of compounds was compared both on a sample-tosample basis and by merging all features from all samples (with duplicates removal based on CAS number). A comparison of the merged compound tables of all samples shows that 


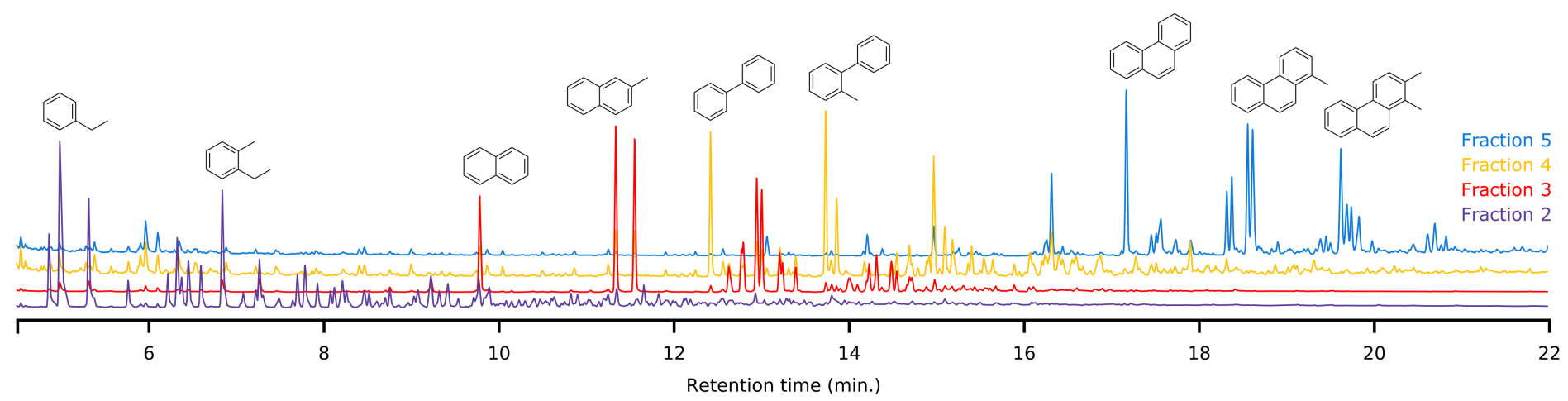

Figure 6 Offset overlay of the GC-MS chromatograms of fractions 2 to 5 obtained using the LC method of a representative sample. Typical compounds in each fraction have been marked to demonstrate the separating power.

Full-size DOI: 10.7717/peerjachem.12/fig-6
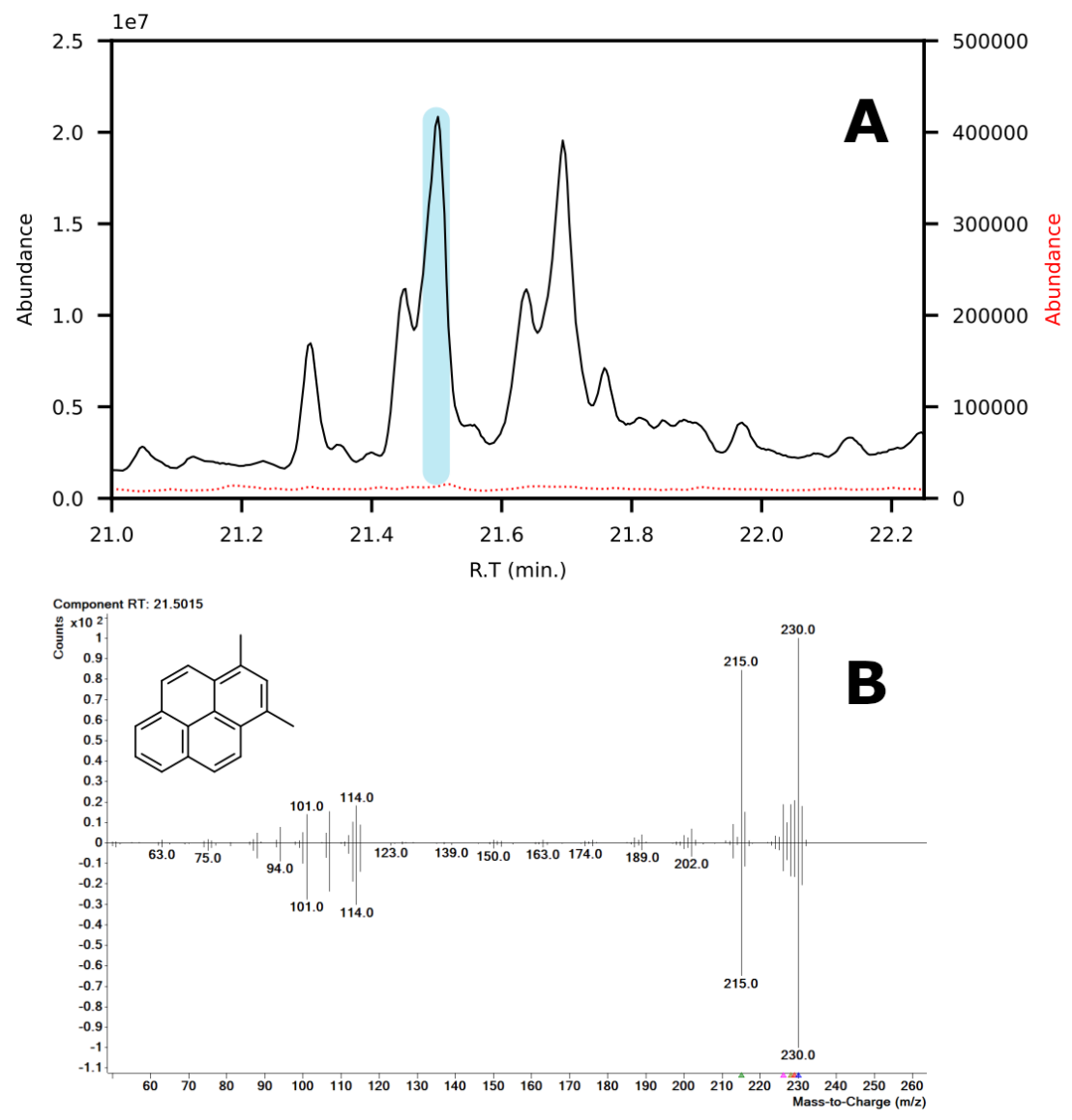

Figure 7 (A) Chromatograms showing the peak of 1,3-dimethylpyrene (marked in blue) found in LCfraction 7 (black, pre-concentrated) and the aromatics fraction obtained via SPE (dotted red). (B) Mass spectra mirror plot of the LC-fraction peak showcasing its purity.

Full-size DOI: 10.7717/peerjachem.12/fig-7 

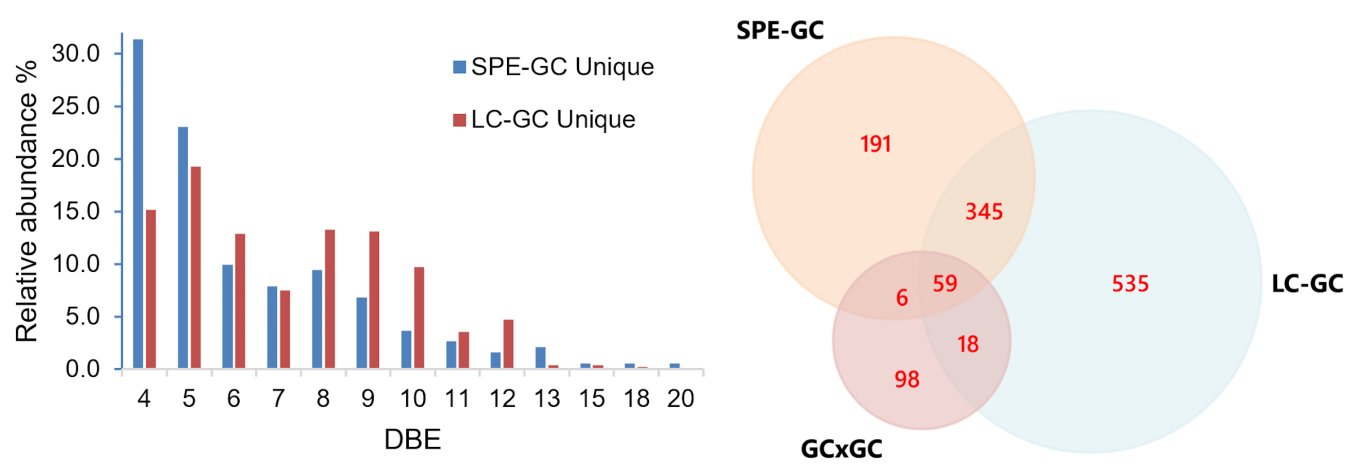

Figure 8 Double bond equivalent (DBE) distribution table for assigned unique compounds obtained from SPE-GC and LC-GC methods. The Venn diagram shows the total amount of assigned compositions obtained from SPE-GC-, LC-GC- and GC $\times$ GC-MS.

Full-size DOI: 10.7717/peerjachem.12/fig-8

using the LC-GC method we can identify 957 compared to 601 compounds using SPE. This is an increase of $37.2 \%$. To increase the match confidence, we applied a retention index (RI) filter, only retaining compounds with a match within 100 units of the library value. By doing so, we identified 426 compared to 300 (42\% increase). This excludes all compounds of which a library RI is not available (approximately $1 \%$ of our feature set). However, a large portion of the compounds only have computationally approximated retention indices and not experimentally determined values. Thus, all filtering and data analyses should be carried out with care and manual intervention. The SPE fraction contains approximately 190 unique compounds with 404 compounds overlapping both analyses. Manual inspection reveals this list contains several petroleum-type compounds and not predominantly background noise or contamination (e.g., plasticizers, column contamination). One plausible source is errors occurring during the automatic processing routines. Small differences in mass spectra (e.g., due to abundance or background level) can lead to closely related library matches being given similar (but different) priority (e.g., isomeric species). Figure 8 shows the DBE distributions of assigned compounds uniquely observed from SPE-GC and LC-GC methods. Noticeably, the DBE distributions are significantly different between the two methods. The distribution of unique compounds from SPE-GC is centered around low DBE 4 and 5 (e.g., monoaromatic), whereas unique compounds from LC-GC are distributed more evenly at higher DBE values. This is expected as the LC-GC method isolates and enriches high aromaticity fractions. These findings showcase the ability of LC-GC as a high-resolution fractionation method for crude oil.

For comparison to comprehensive multi-dimensional chromatography, the samples were analyzed by our in-house routine GC $\times$ GC-MS method (i.e., solvent dilution, filtration and analysis) (Fig. 9). The objective of the GC $\times$ GC method is not to maximize feature ID but enable multi-class analysis/fingerprinting with minimal to no sample preparation. Furthermore, the SPE-LC-GC and GC $\times$ GC analyses were carried out on different instruments which makes direct comparison challenging. For GC $\times \mathrm{GC}$ an Agilent 7200B QTOF high-resolution mass spectrometer was used. For SPE-LC-GC, an Agilent 

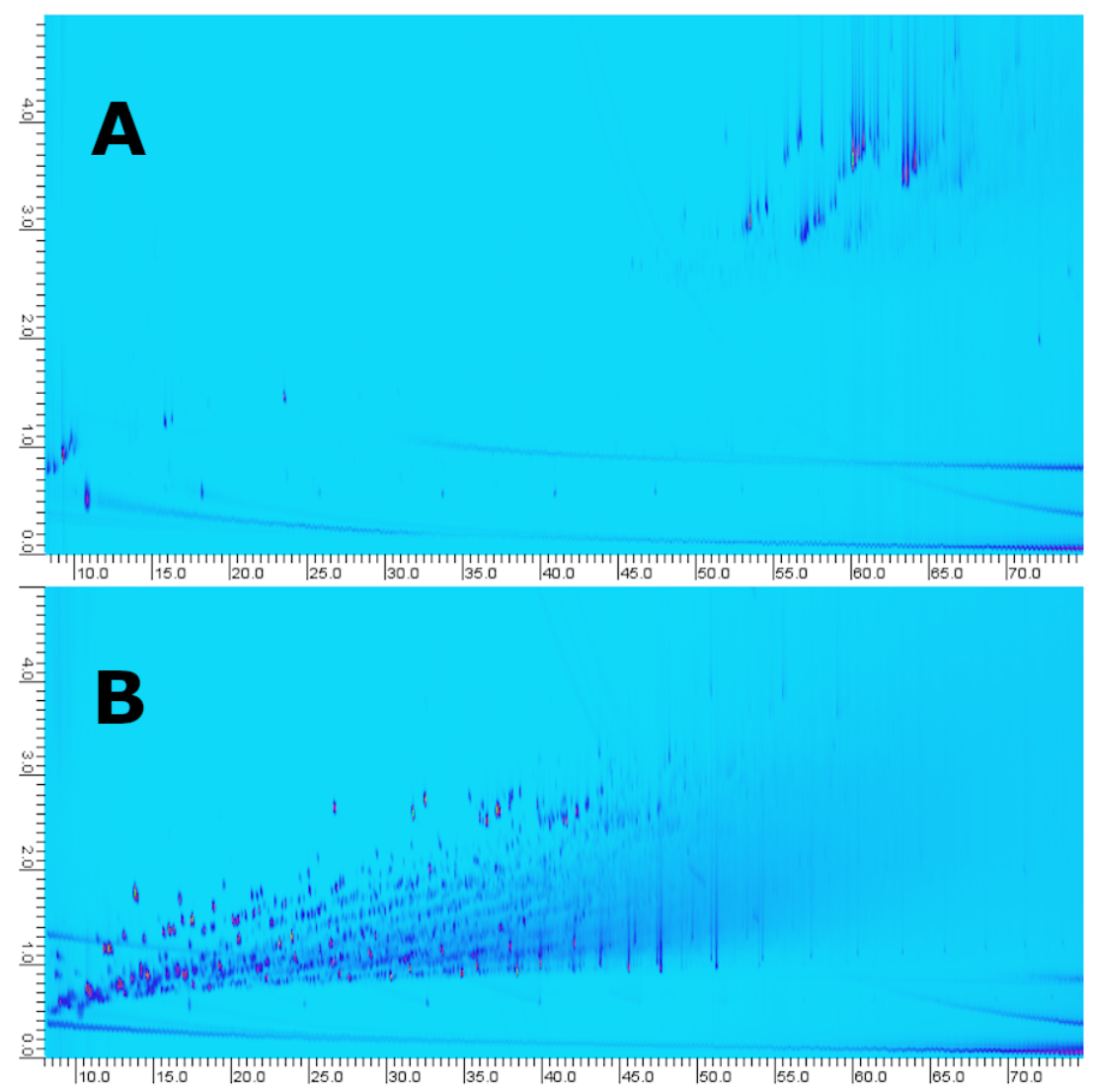

Figure 9 GCx GC-Chromatograms of fraction 5 obtained using the LC-method (A) compared to whole oil analysis (B, i.e., no pre-fractionation). The LC-fraction has a high abundance of alkyl phenanthrene isomers at or below the LOD seen in whole oil GC $\times$ GC-MS.

Full-size DOI: 10.7717/peerjachem.12/fig-9

5977B single quadrupole equipped with a High-Efficiency Source (HES) was used. The HES has both higher sensitivity and dynamic range. Secondly, for GC $\times$ GC, the dilution factor was adjusted so that the analytes with the highest abundance were at detector saturation. Here, we see that although GC $\times$ GC is not restricted in terms of peak capacity, it does fall short in terms of dynamic range. After blob detection, library matching and filtering we obtain 63 tentative hits in a single sample. With corresponding processing settings, we identified 143 compounds by multi-fraction LC-GC-MS analysis of the same sample. This is an increase of $127 \%$. In terms of manual intervention, ease of use and time of analysis, GC $\times$ GC is preferred compared to LC-GC-MS. However, the amount of data generated using the latter is more comprehensive in our case.

A Venn diagram was constructed to compare three methods (Fig. 8). The number within each colored circle represents the number of assigned unique compounds for each method, whereas numbers in overlapped zones represent the number of compounds that have been co-assigned from corresponding methods. The amount of compositions obtained from LCxGC (957) significantly surpasses GC $\times$ GC (181) and SPE-GC (601). We obtained approximately $50 \%$ unique compounds with GC $\times$ GC and LC-GC and 32\% with SPE-GC. 
Table 3 Comparison of the number of tentatively identified species in SPE-GC and GCGC versus LCGC. The calculations are based on merged data of all samples with the removal of duplicates. Unique indicates compounds not identified in the compared methods.

\begin{tabular}{lllllll} 
Filter & $\begin{array}{l}\text { GCxGC } \\
\text { Tot. }\end{array}$ & $\begin{array}{l}\text { GCxGC } \\
\text { Unique }\end{array}$ & $\begin{array}{l}\text { SPE-GC } \\
\text { Tot. }\end{array}$ & $\begin{array}{l}\text { SPE-GC } \\
\text { Unique }\end{array}$ & $\begin{array}{l}\text { LC-GC } \\
\text { Tot. }\end{array}$ & $\begin{array}{l}\text { LC-GC } \\
\text { Unique }\end{array}$ \\
\hline No formula or R.I filter & 181 & 98 & 601 & 191 & 957 & 535 \\
No formula filter, R.I \pm 100 units & 82 & 15 & 300 & 68 & 426 & 187 \\
N,S,O $\geq 1$, no R.I filter & 80 & 79 & 311 & 151 & 517 & 357 \\
N,S,O $\geq 1$, R.I \pm 100 units & 6 & 6 & 91 & 44 & 124 & 81 \\
\hline
\end{tabular}

The Venn diagram also shows that co-assigned compounds of those three methods cover a narrow range of overall chemical composition (59 co-assigned compounds) of crude oil. Again, it is worth noting that there are differences in terms of dilution factor and instrumental parameters for those methods. Therefore, the comparison is biased but still relevant to evaluate the LC-GC method for trace components analysis.

\section{CONCLUSIONS}

We have developed a method for high-resolution fractionation of complex crude oil matrices. By using sub-micron LC columns we obtained high efficiency and resolution which allowed intra-class compound separation. This is in contrast with traditional methods, e.g., SPE, which yields a single aromatics fraction. The method is especially advantageous for the isolation of trace species. Multiple compounds not observed by SPE-GC-MS were pre-concentrated yielding high abundance and spectral quality. The increase in the number of tentatively identified peaks is thus a result of both reduced co-elution and an increase in analyte signal-to-noise ratio.

By characterization of 14 crude oils, we extended the identification to a large number of hydrocarbon and N,S,O-containing aromatics. Of the 517 uniquely identified compounds, $69 \%$ (357) contain either N,S,O (or a combination of) atoms (Table 3). The structures of five representative compounds are presented in Fig. 10. Aromatic nitrogen and sulfur compounds are detrimental in petroleum processing. Furthermore, they potentially have biological activity and may pose an environmental and toxicological hazard (López García et al., 2002; Anyanwu \& Semple, 2015; Zhang et al., 2018; Vetere, Pröfrock \& Schrader, 2021). Therefore, their characterization is an important pursuit. They are routinely analyzed by direct infusion mass spectrometry that provides the molecular formula but not connectivity (Guan, Marshall \& Scheppele, 1996; Purcell et al., 2007b; Purcell et al., 2007a; Corilo, Rowland \& Rodgers, 2016). Thus, isolation and GC-MS analysis with library matching provide valuable information on their presence in oil samples.

The relatively long fractionation time $(60 \mathrm{~min})$ and the number of fractions generated lead to a full sample analysis time of $6 \mathrm{~h}$ (when characterizing the first 7 fractions using GC-MS). Several steps require manual intervention, i.e., dilution and pre-concentration of fractions and moving the samples from the LC to the GC. It would therefore be beneficial to implement more automation, e.g., by using liquid handling robotics (ultimately with direct hyphenation to the GC). We observed minor co-elution during the analysis of 
<smiles>O=C(O)c1ccc2c(c1)CCC2</smiles>

Indan-5-carboxylic acid Fraction 2<smiles>Nc1cccc2ccccc12</smiles>

1-Naphthylamine Fraction 3

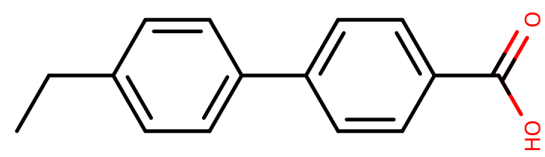

4'-Ethyl-4-biphenylcarboxylic acid Fraction 4

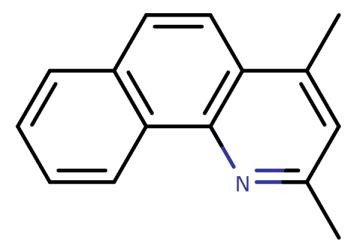

2,4-Dimethylbenzo[h]quinoline Fraction 5

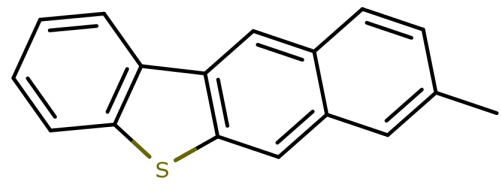

8-Methyl-benzo[b]naphtho[2,3-d]thiophene Fraction 6

Figure 10 The molecular structures of five unique compounds identified in separate fractions (2 to 6) obtained by using the LC-method.

Full-size DOI: 10.7717/peerjachem.12/fig-10

latter fractions. Combining the LC-fractionation with subsequent GC $\times$ GC analysis would increase the power of the method further. However, it would require an intense data processing workflow with high demands in computational power. Something that is already challenging in comprehensive GC $\times$ GC studies (Reichenbach et al., 2019; Wilde et al., 2020; Stefanuto, Smolinska \& Focant, 2021).

\section{ACKNOWLEDGEMENTS}

The authors are grateful for the donation of the samples from Total E\&P Denmark. Furthermore, the authors wish to thank Karen L. Feilberg for discussions.

\section{ADDITIONAL INFORMATION AND DECLARATIONS}

\section{Funding}

This work was supported by the Danish Hydrocarbon Research and Technology Center. The funders had no role in study design, data collection and analysis, decision to publish, or preparation of the manuscript.

\section{Grant Disclosures}

The following grant information was disclosed by the authors:

Danish Hydrocarbon Research and Technology Center.

\section{Competing Interests}

The authors declare there are no competing interests. 


\section{Author Contributions}

- Khoa Huynh conceived and designed the experiments, performed the experiments, analyzed the data, prepared figures and/or tables, authored or reviewed drafts of the paper, and approved the final draft.

- Annette E. Jensen conceived and designed the experiments, performed the experiments, authored or reviewed drafts of the paper, and approved the final draft.

- Jonas Sundberg conceived and designed the experiments, performed the experiments, analyzed the data, performed the computation work, prepared figures and/or tables, authored or reviewed drafts of the paper, and approved the final draft.

\section{Data Availability}

The following information was supplied regarding data availability:

The raw data (GC-MS, feature tables) are available at Zenodo: Sundberg, Jonas. (2021). Extended characterization of petroleum aromatics by off-line LC-GC-MS (dataset) [Data set]. Zenodo. https://doi.org/10.5281/zenodo.5121065.

\section{Supplemental Information}

Supplemental information for this article can be found online at http://dx.doi.org/10.7717/ peerj-achem.12\#supplemental-information.

\section{REFERENCES}

Aftalion F. 2001. A history of the international chemical industry. Philadelphia: Chemical Heritage Press.

Alzaga R, Montuori P, Ortiz L, Bayona JM, Albaigés J. 2004. Fast solid-phase extractiongas chromatography-mass spectrometry procedure for oil fingerprinting. Journal of Chromatography A 1025:133-138 DOI 10.1016/j.chroma.2003.10.080.

Andersson JT, Achten C. 2015. Time to say goodbye to the 16 EPA PAHs? Toward an up-to-date use of PACs for environmental purposes. Polycyclic Aromatic Compounds 35:330-354 DOI 10.1080/10406638.2014.991042.

Anyanwu IN, Semple KT. 2015. Fate and behaviour of nitrogen-containing polycyclic aromatic hydrocarbons in soil. Environmental Technology \& Innovation 3:108-120 DOI 10.1016/j.eti.2015.02.006.

Berrueta LA, Gallo B, Vicente F. 1995. A review of solid phase extraction: basic principles and new developments. Chromatographia 40:474-483 DOI 10.1007/BF02269916.

Buszewski B, Szultka M. 2012. Past, present, and future of solid phase extraction: a review. Critical Reviews in Analytical Chemistry 42:198-213

DOI 10.1080/07373937.2011.645413.

Carvalho Dias L, Bomfim Bahia PV, Nerydo Amaral D, Machado ME. 2020. Nitrogen compounds as molecular markers: an overview of analytical methodologies for its determination in crude oils and source rock extracts. Microchemical Journal 157:105039 DOI 10.1016/j.microc.2020.105039. 
Corilo YE, Rowland SM, Rodgers RP. 2016. Calculation of the total sulfur content in crude oils by positive-ion atmospheric pressure photoionization fourier transform ion cyclotron resonance mass spectrometry. Energy \& Fuels 30:3962-3966 DOI 10.1021/acs.energyfuels.6b00497.

Countryman S, Kelly K, Garriques M. 2005. An improved method for diesel fractionation following the revised Massachusetts EPH guidelines. Application note TN-2022. Torrance: Phenomenex Inc..

Davis JM, Giddings JC. 1983. Statistical theory of component overlap in multicomponent chromatograms. Analytical Chemistry 55:418-424 DOI 10.1021/ac00254a003.

Gilgenast E, Boczkaj G, Przyjazny A, Kamiński M. 2011. Sample preparation procedure for the determination of polycyclic aromatic hydrocarbons in petroleum vacuum residue and bitumen. Analytical and Bioanalytical Chemistry 401:1059-1069 DOI 10.1007/s00216-011-5134-9.

Guan S, Marshall AG, Scheppele SE. 1996. Resolution and chemical formula identification of aromatic hydrocarbons and aromatic compounds containing sulfur, nitrogen, or oxygen in petroleum distillates and refinery streams. Analytical Chemistry 68:46-71 DOI 10.1021/ac9507855.

International Energy Agency (IEA). 2021. Net Zero by 2050: A roadmap for the global energy sector. Paris: IEA. Available at https://www.iea.org/reports/net-zero-by-2050.

Jennerwein MK, Eschner M, Gröger T, Wilharm T, Zimmermann R. 2014. Complete group-type quantification of petroleum middle distillates based on comprehensive two-dimensional gas chromatography time-of-flight mass spectrometry (GC $\times$ GC-TOFMS) and visual basic scripting. Energy \& Fuels 28:5670-5681 DOI 10.1021/ef501247.

John GF, Han Y, Clement TP. 2016. Weathering patterns of polycyclic aromatic hydrocarbons contained in submerged Deepwater Horizon oil spill residues when re-exposed to sunlight. Science of The Total Environment 573:189-202 DOI 10.1016/j.scitotenv.2016.08.059.

Keith LH. 2015. The Source of US EPA's sixteen PAH priority pollutants. Polycyclic Aromatic Compounds 35:147-160 DOI 10.1080/10406638.2014.892886.

Krajewski LC, Rodgers RP, Marshall AG. 2017. 126264 assigned chemical formulas from an atmospheric pressure photoionization 9.4 T Fourier transform positive ion cyclotron resonance mass spectrum. Analytical Chemistry 89:11318-11324 DOI 10.1021/acs.analchem.7b02004.

Lawal AT. 2017. Polycyclic aromatic hydrocarbons. A review. Cogent Environmental Science 3:1339841 DOI 10.1080/23311843.2017.1339841.

López García C, Becchi M, Grenier-Loustalot MF, Païsse O, Szymanski R. 2002. Analysis of Aromatic sulfur compounds in gas oils using GC with sulfur chemiluminescence detection and high-resolution MS. Analytical Chemistry 74:3849-3857 DOI 10.1021/ac011190.

Marshall AG, Rodgers RP. 2008. Petroleomics: chemistry of the underworld. Proceedings of the National Academy of Sciences of the United States of America 105:18090-18095 DOI 10.1073/pnas.0805069105. 
Mössner SG, Wise SA. 1999. Determination of polycyclic aromatic sulfur heterocycles in fossil fuel-related samples. Analytical Chemistry 71:58-69 DOI 10.1021/ac980664f.

Nizio KD, McGinitie TM, Harynuk JJ. 2012. Comprehensive multidimensional separations for the analysis of petroleum. Journal of Chromatography A 1255:12-23 DOI 10.1016/j.chroma.2012.01.078.

Palacio Lozano DC, Gavard R, Arenas-Diaz JP, Thomas MJ, Stranz DD, MejíaOspino E, Guzman A, Spencer SEF, Rossell D, Barrow MP. 2019. Pushing the analytical limits: new insights into complex mixtures using mass spectra segments of constant ultrahigh resolving power. Chemical Science 10:6966-6978 DOI 10.1039/C9SC02903F.

Pampanin DM, Sydnes MO (eds.) 2017. Petrogenic polycyclic aromatic hydrocarbons in the aquatic environment: analysis, synthesis, toxicity and environmental impact. Bentham Science Publishers DOI 10.2174/97816810842751170101.

Pillai I, Ritchie L, Heywood R, Wilson G, Pahlavanpour B, Setford S, Saini S. 2005. Development of an improved analytical method for the determination of carcinogenic polycyclic aromatic hydrocarbons in transformer oil. Journal of Chromatography $A$ 1064:205-212 DOI 10.1016/j.chroma.2004.12.066.

Poulsen KG, Kristensen M, Tomasi G, Cruz MDela, Christensen JH. 2018. The pixel-based chemometric approach for oil spill identification and hydrocarbon source differentiation. In: Stout SA, Wang Z, eds. Oil spill environmental forensics case studies. Oxford: Butterworth-Heinemann, 443-463 DOI 10.1016/B978-0-12-804434-6.00021-5.

Purcell JM, Juyal P, Kim D-G, Rodgers RP, Hendrickson CL, Marshall AG. 2007a. Sulfur speciation in petroleum: atmospheric pressure photoionization or chemical derivatization and electrospray ionization fourier transform ion cyclotron resonance mass spectrometry. Energy \& Fuels 21:2869-2874 DOI 10.1021/ef700210.

Purcell JM, Rodgers RP, Hendrickson CL, Marshall AG. 2007b. Speciation of nitrogen containing aromatics by atmospheric pressure photoionization or electrospray ionization fourier transform ion cyclotron resonance mass spectrometry. Journal of the American Society for Mass Spectrometry 18:1265-1273 DOI 10.1016/j.jasms.2007.03.030.

Reichenbach SE, Tao Q, Cordero C, Bicchi C. 2019. A data-challenge case study of analyte detection and identification with comprehensive two-dimensional gas chromatography with mass spectrometry (GC $\times$ GC-MS). Separations 6:38 DOI 10.3390/separations6030038.

Requejo AG, Sassen R, McDonald T, Denoux G, Kennicutt MC, Brooks JM. 1996. Polynuclear aromatic hydrocarbons (PAH) as indicators of the source and maturity of marine crude oils. Organic Geochemistry 24(1):1017-1033 DOI 10.1016/S0146-6380(96)00079-4.

Rosenthal D. 1982. Theoretical limitations of gas chromatographic/mass spectrometric identification of multicomponent mixtures. Analytical Chemistry 54:63-66 DOI 10.1021/ac00238a020. 
Stefanuto P-H, Smolinska A, Focant J-F. 2021. Advanced chemometric and data handling tools for GC $\times$ GC-TOF-MS. Trends in Analytical Chemistry 139:116251 DOI 10.1016/j.trac.2021.116251.

Stilo F, Bicchi C, Jimenez-Carvelo AM, Cuadros-Rodriguez L, Reichenbach SE, Cordero C. 2021. Chromatographic fingerprinting by comprehensive twodimensional chromatography: Fundamentals and tools. Trends in Analytical Chemistry 134:116133 DOI 10.1016/j.trac.2020.116133.

Stout SA, Emsbo-Mattingly SD, Douglas GS, Uhler AD, McCarthy KJ. 2015. Beyond 16 priority pollutant PAHs: a review of PACs used in environmental forensic chemistry. Polycyclic Aromatic Compounds 35:285-315 DOI 10.1080/10406638.2014.891144.

Sundberg J, Feilberg KL. 2020. Characterization of heteroatom distributions in the polar fraction of North Sea oils using high-resolution mass spectrometry. Journal of Petroleum Science and Engineering 184:106563 DOI 10.1016/j.petrol.2019.106563.

Vetere A, Pröfrock D, Schrader W. 2021. Qualitative and quantitative evaluation of sulfur-containing compound types in heavy crude oil and its fractions. Energy \& Fuels 35:8723-8732 DOI 10.1021/acs.energyfuels.1c00491.

Wang Z, Fingas M, Li K. 1994. Fractionation of a light crude oil and identification and quantitation of Aliphatic, Aromatic, and biomarker compounds by GC-FID and GC-MS, Part I. Journal of Chromatographic Science 32:361-366 DOI 10.1093/chromsci/32.9.361.

Wei O, Xu X, Zhang Y, Yang B, Ye Q, Yang Z. 2018. Multidimensional gas chromatography-mass spectrometry method for fingerprinting polycyclic aromatic hydrocarbons and their alkyl-homologs in crude oil. Analytical Letters 51:483-495 DOI 10.1080/00032719.2017.1341905.

Wilde MJ, Zhao B, Cordell RL, Ibrahim W, Singapuri A, Greening NJ, Brightling CE, Siddiqui S, Monks PS, Free RC. 2020. Automating and extending comprehensive two-dimensional gas chromatography data processing by interfacing open-source and commercial software. Analytical Chemistry 92:13953-13960 DOI 10.1021/acs.analchem.0c02844.

Wilton NM, Wise SA, Robbat A. 2017. Errors in alkylated polycyclic aromatic hydrocarbon and sulfur heterocycle concentrations caused by currently employed standardized methods. Analytica Chimica Acta 977:20-27 DOI 10.1016/j.aca.2017.04.017.

Yadav VG, Yadav GD, Patankar SC. 2020. The production of fuels and chemicals in the new world: critical analysis of the choice between crude oil and biomass vis-à-vis sustainability and the environment. Clean Technologies and Environmental Policy 22:1757-1774 DOI 10.1007/s10098-020-01945-5.

Zhang G, Yang C, Serhan M, Koivu G, Yang Z, Hollebone B, Lambert P, Brown CE. 2018. Characterization of nitrogen-containing polycyclic aromatic heterocycles in crude oils and refined petroleum products. In: Advances in marine biology. Elsevier, 59-96 DOI 10.1016/bs.amb.2018.09.006.

Zhao Y, Hong B, Fan Y, Wen M, Han X. 2014. Accurate analysis of polycyclic aromatic hydrocarbons (PAHs) and alkylated PAHs homologs in crude oil for improving 
the gas chromatography/mass spectrometry performance. Ecotoxicology and Environmental Safety 100:242-250 DOI 10.1016/j.ecoenv.2013.10.018. 